\title{
Nivel de riesgo y casos incidentes de úlceras por presión en pacientes hospitalizados en los servicios de medicina de un Hospital Nacional
}

\author{
Cesar Zuñiga-Castro ${ }^{1 a}$, Nadia Abigail Espinoza-Cáceres ${ }^{2 a}$, Maria Ángela Fernandez-Pacheco ${ }^{3 b}$
}

\begin{abstract}
RESUMEN
Objetivo: determinar el nivel de riesgo e incidencia de úlceras por presión en pacientes hospitalizados. Material y métodos se desarrolló el estudio de tipo descriptivo prospectivo longitudinal de tendencia, en los servicios de medicina del Hospital Arzobispo Loayza en una población de 151 pacientes quienes fueron seleccionados de forma aleatoria utilizando como criterios de inclusión, el déficit de movimiento y la alteración de conciencia, alteración en el control de esfinteres y alteración de la nutrición. La técnica utilizada fue la observacional, la cual se realizó diariamente valorando el estado del paciente, tanto en el momento del ingreso como durante su estancia hospitalaria; como instrumento se utilizó una lista de chequeo elaborada considerando la escala de valoración de riesgo de úlceras por presión ESCALA DE NORTON para poder evaluar el nivel de riesgo de los pacientes. Para medir la variable incidencia de úlceras por presión se utilizó la fórmula estadística probabilística. Para determinar el nivel de riesgo y la incidencia de úlceras por presión se aplicó la prueba estadística de X2. RESULTADOS: muestran que el nivel de riesgo a úlceras por presión encontrado en la población de pacientes hospitalizados en el servicio de medicina, fue de Alta a Muy alta y en las dimensiones estado físico, actividad incontinencia reporto un estado regular.
\end{abstract}

Palabras claves: Ulcera por presión, enfermeria, pacientes internados, adulto mayor.

\section{RISK LEVEL AND INCIDENT CASES OF PRESSURE ULCERS IN HOSPITALIZED PATIENTS AT A MEDICINE UNIT FROM A PUBLIC HOSPITAL}

\begin{abstract}
Objective: The aim of the project was to determine the risk level and incident of pressure ulcers. Material and methods: A prospective, longitudinal, descriptive study was conducted at Arzobispo Loayza Hospital's Medicine unit. A random sample of 151 who fulfilled the inclusion criteria, movement deficit and altered state of consciousness, alteration in sphincter control and nutrition.

An observational technique was used every day to assess the patients during admission and hospital stay. A check list created by the researchers considering the The Norton Scale for Predicting Pressure Ulcer Risk.

A probability statistical analysis of the quantitative data was conducted. The $\mathbf{X}^{2}$ statistical test was used to determine the risk level and incident cases of pressure ulcers.

Results: The pressure ulcers risk level found in the medicine unit's hospitalized patients was high and very high; in physical state dimension and incontinence activity the state was regular.
\end{abstract}




\section{INTRODUCCION}

"La úlcera por presión (UPP), es una lesión de origen isquémico, localizada en la piel y tejidos subyacentes con pérdida de sustancia cutánea producida por presión prolongada o fricción entre dos planos duros" (1). Constituye un tipo especial de lesiones causadas por un trastorno de irrigación sanguínea y nutrición tisular como resultado de la presión prolongada sobre prominencias óseas o cartilaginosas. Las lesiones no solo se producen en posición supina, sino también en los pacientes confinados a la silla de ruedas. Las UPP constituyen un importante problema de salud que afecta a todos los sistemas sanitarios desarrollados.

Las úlceras por presión pueden causar infecciones graves, algunas de las cuales pueden poner la vida en peligro. Pueden constituir un problema para las personas en los centros de cuidados especializados por lo que la prevención de las UPP es el eje fundamental en todos los niveles asistenciales.

Ocurren con mayor frecuencia en la edad avanzada, en pacientes confinados en cama o a silla de ruedas, desnutridos, comprometidos de conciencia y mal apoyo familiar, siendo los sitios más comprometidos en más del $80 \%$ de los casos el sacro, trocánteres, nalgas, maléolos externos y talones. La valoración del riesgo es un aspecto clave en la prevención.

La razón de la valoración del riesgo es la identificación de los individuos que necesitan medidas de prevención y la identificación de los factores específicos que los ponen en situación de riesgo. Para ello se utilizan las escalas de valoración del riesgo. Las escalas sirven para valorar el riesgo que tiene una persona de desarrollar UPP, con el objeto de poder determinar un plan de cuidados preventivos (2). Entre ellas se encuentra la Escala desarrollada por NORTON en 1962, la cual es aplicada en pacientes geriátricos. Esta herramienta incluye 5 aspectos a valorar: estado físico general (nutrición, ingesta de líquidos, temperatura corporal e hidratación), estado mental, actividad, movilidad e incontinencia (3).

La Escala de NORTON mide el riesgo que tiene un paciente de padecer úlceras por presión. Su utilización ha permitido valorar los riesgos en el paciente, determinar las intervenciones para la posterior evaluación de los resultados.

Según los datos estadístico, las causas más frecuentes de morbilidad en hospitalización en pacientes mayores de 60 años en los servicios de medicina son insuficiencia cardiaca congestiva, accidente cerebro vascular, neumonía no especificada, enfermedad renal crónica y diabetes mellitus; identificándose un promedio de 10 pacientes en riesgo de presentar UPP al mes durante el periodo de hospitalización, donde aproximadamente el $70 \%$ de todas las UPP ocurren en personas mayores de 70 años de edad (4).

El objetivo del estudio fue determinar el nivel de riesgo y casos incidentes de úlceras por presión en pacientes hospitalizados de los servicios de medicina de un hospital nacional.

\section{MATERIAL Y MÉTODOS}

El estudio fue descriptivo transversal, con una población total de 620 pacientes y una muestra de 151 pacientes cuyos criterios de inclusión fueron, ser pacientes hasta las 24 horas de ingreso al servicio, con déficit de movimiento, con alteración de conciencia, alteración en el control de esfínteres, alteración de la nutrición, y con grado de dependencia III; excluyéndose a pacientes que al momento del ingreso presenten UPP en cualquier región del cuerpo $\mathrm{y}$ de cualquier estadio.

Para la recolección de datos se utilizó la técnica observacional al momento del ingreso y durante su estancia hospitalaria aplicándose la guía de observación para registrar los cambios físicos en las zonas de presión, a su vez empleamos la escala de Norton para poder evaluar el nivel de riesgo que tienen los pacientes, tanto al ingreso como estando ya hospitalizados, esta herramienta valoró cinco parámetros y tiene un puntaje mínimo de cinco y máximo de veinte puntos.

Previo a la recolección de datos se solicitó la autorización por parte del Comité Institucional de Ética (CIE) de la Universidad Peruana Cayetano Heredia y del Hospital Nacional.

Los investigadores identificaron a los pacientes de ingreso diario que cumplían con los criterios de inclusión informándoseles acerca del proyecto de investigación utilizando una hoja informativa. Una vez que aceptaron participar se realizó la primera observación diaria, en cinco días por paciente, las cuales se registran en dicha guía, identificándose el nivel de riesgo a desarrollar UPP según Escala Norton. Los datos se recogieron de octubre a diciembre del año 2013.

Para el procesamiento y análisis de datos, se introdujeron en la base datos diseñados en el programa Excel. Los resultados se presentan en tablas estadísticas de doble entrada. 


\section{RESULTADOS}

Tabla 1: Nivel de riesgo e incidencia de ulceras por presión según escala de Norton en pacientes hospitalizados en los servicios de medicina de un hospital nacional, 2013

\begin{tabular}{|c|c|l|c|}
\hline \multirow{2}{*}{ Nivel de riesgo } & \multicolumn{2}{|c|}{ Incidencia de UPP } & $\begin{array}{c}\text { Total } \\
\text { General }\end{array}$ \\
\hline & NO & SI & \\
\hline Mínimo & 2.65 & 0.00 & 2.65 \\
\hline Medio & 10.60 & 0.66 & 11.26 \\
\hline Alto & 60.26 & 7.29 & 67.55 \\
\hline Muy Alto & 14.57 & 3.97 & 18.54 \\
\hline Total general & 88.08 & 11.92 & 100.00 \\
\hline
\end{tabular}

Se observa que el $98.68 \%$ obtuvieron un estado físico general Regular, de los cuales el $66.89 \%$ alcanzo un nivel de riesgo Alto.

Tabla 2: Nivel de riesgo en la dimensión estado físico general según escala de Norton, 2013

\begin{tabular}{|c|c|c|c|c|c|}
\hline $\begin{array}{c}\text { Nivel de } \\
\text { riesgo }\end{array}$ & \multicolumn{4}{|c|}{ Estado Físico General } & $\begin{array}{c}\text { Total } \\
\text { General }\end{array}$ \\
\hline & Bueno & Mediano & Regular & Muy Malo & \\
\hline Mínimo & 0 & 0 & 2.65 & 0 & 2.65 \\
\hline Medio & 0 & 0.66 & 10.60 & 0 & 11.26 \\
\hline Alto & 0 & 0.66 & 66.89 & 0 & 67.55 \\
\hline Muy Alto & 0 & 0 & 18.54 & 0 & 18.54 \\
\hline $\begin{array}{c}\text { Total } \\
\text { General }\end{array}$ & 0 & 1.32 & 98.68 & 0 & 100.00 \\
\hline
\end{tabular}

Según tabla 2, De la población en estudio el 67.55\% (102) alcanzó un nivel de riesgo alto, de los cuales el 53.64\% (81) presento estado mental apático, en la dimensión estado mental.
Tabla 3: Nivel de riesgo en la dimensión estado mental según escala de Norton, 2013

\begin{tabular}{|c|c|c|c|c|c|}
\hline Nivel de & \multicolumn{4}{|c|}{ Estado mental } & \multirow{2}{*}{$\begin{array}{c}\text { Total } \\
\text { general }\end{array}$} \\
\hline & Alerta & Apático & Confuso & $\begin{array}{c}\text { Estuporoso/ } \\
\text { Comatoso }\end{array}$ & \\
\hline Mínimo & 0.66 & 1.99 & 0.00 & 0.00 & 2.65 \\
\hline Medio & 0.66 & 10.60 & 0.00 & 0.00 & 11.26 \\
\hline Alto & 0.66 & 53.64 & 13.25 & 0.00 & 67.55 \\
\hline Muy Alto & 0.00 & 1.99 & 9.27 & 7.28 & 18.54 \\
\hline $\begin{array}{c}\text { Total } \\
\text { general }\end{array}$ & 1.99 & 68.21 & 22.52 & 7.28 & 100.00 \\
\hline
\end{tabular}

En la tabla 3, el 80.79\% fue catalogada con una Actividad Muy Limitada.

Tabla 4: Nivel de riesgo en la dimensión actividad según escala Norton, 2013

\begin{tabular}{|c|c|c|c|c|c|}
\hline $\begin{array}{c}\text { Nivel de } \\
\text { riesgo }\end{array}$ & \multicolumn{4}{|c|}{ Actividad } & $\begin{array}{c}\text { Total } \\
\text { General }\end{array}$ \\
\hline & Ambulante & $\begin{array}{c}\text { Dismi- } \\
\text { nuida }\end{array}$ & $\begin{array}{c}\text { Muy } \\
\text { Limitada }\end{array}$ & Inmóvil & \\
\hline Mínimo & 0.00 & 2.65 & 0.00 & 0.00 & 2.65 \\
\hline Medio & 0.00 & 5.96 & 5.30 & 0.00 & 11.26 \\
\hline Alto & 0.00 & 1.99 & 65.56 & 0.00 & 67.55 \\
\hline Muy Alto & 0.00 & 0.00 & 9.93 & 8.61 & 18.54 \\
\hline Total & 0.00 & 10.60 & 80.79 & 8.61 & 100.00 \\
\hline General & & & & & \\
\hline
\end{tabular}

Según tabla 4 , el $70.86 \%$ obtuvo una clasificación de Sentado en la dimensión Movilidad. 
Tabla 5: Nivel de riesgo en la dimensión movilidad según escala Norton, 2013

\begin{tabular}{|c|c|c|c|c|c|}
\hline $\begin{array}{c}\text { Nivel de } \\
\text { riesgo }\end{array}$ & \multicolumn{4}{|c|}{ Movilidad } & $\begin{array}{c}\text { Total } \\
\text { General }\end{array}$ \\
\hline & Total & $\begin{array}{c}\text { Camina con } \\
\text { Ayuda }\end{array}$ & Sentado & Encamado & \\
\hline Mínimo & 0.00 & 2.65 & 0.00 & 0.00 & 2.65 \\
\hline Medio & 0.00 & 4.64 & 6.62 & 0.00 & 11.26 \\
\hline Alto & 0.00 & 0.00 & 61.59 & 5.96 & 67.55 \\
\hline Muy Alto & 0.00 & 0.00 & 2.65 & 15.89 & 18.54 \\
\hline $\begin{array}{c}\text { Total } \\
\text { General }\end{array}$ & 0.00 & 7.29 & 70.86 & 21.85 & 100.00 \\
\hline
\end{tabular}

El $60.26 \%$ obtuvo una clasificación de incontinencia permanente, ya sea Urinaria o Fecal.

Tabla 6: Nivel de riesgo en la dimensión incontinencia según escala Norton, 2013

\begin{tabular}{|c|c|c|c|c|c|}
\hline \multirow{2}{*}{$\begin{array}{l}\text { Nivel de } \\
\text { riesgo }\end{array}$} & \multicolumn{4}{|c|}{ Incontinencia } & \multirow{2}{*}{$\begin{array}{c}\text { Total } \\
\text { Gene- } \\
\text { ral }\end{array}$} \\
\hline & $\begin{array}{c}\text { Ningu- } \\
\text { na }\end{array}$ & Ocasional & $\begin{array}{c}\text { Urinaria } \\
\text { o Fecal }\end{array}$ & $\begin{array}{c}\text { Urinaria y } \\
\text { Fecal }\end{array}$ & \\
\hline Mínimo & 1.32 & 1.32 & 0.00 & 0.00 & 2.65 \\
\hline Medio & 4.64 & 5.96 & 0.66 & 0.00 & 11.26 \\
\hline Alto & 0.66 & 13.25 & 52.98 & 0.66 & 67.55 \\
\hline Muy Alto & 0.00 & 0.00 & 6.62 & 11.92 & 18.54 \\
\hline $\begin{array}{c}\text { Total } \\
\text { General }\end{array}$ & 6.62 & 20.53 & 60.26 & 12.58 & 100.00 \\
\hline
\end{tabular}

Según tabla 6, muestra que del total de pacientes que desarrollaron úlceras por presión en el periodo de estudio 11 , estaban con un nivel de riesgo alto y 6 muy alto.
Tabla 7. Frecuencia de ulceras por presión en los pacientes hospitalizados en el servicio de medicina del hospital nacional 2013

\begin{tabular}{|c|c|}
\hline Nivel de riesgo & Pacientes con upp \\
\hline Mínimo & 0 \\
\hline Medio & 1 \\
\hline Alto & 11 \\
\hline Muy Alto & 6 \\
\hline TOTAL GENERAL & 18 \\
\hline
\end{tabular}

Se encontró que del total de población sujeto de estudio en su mayoría se encontraron con un nivel de riesgo a ulcera por presión alto.

\section{DISCUSION}

Con el objetivo de determinar el nivel de riesgo y la incidencia de úlceras por presión en los pacientes de los servicios de medicina en un hospital nacional, se desarrolló el estudio, encontrándose en los resultados que de la población sujeto de investigación, un porcentaje considerable tuvo alto riesgo a desarrollar UPP, situación condicionado probablemente por el tipo de población encontrada, siendo en su mayoría población geriátrica con afecciones neurologías metabólicas y otras cuya condición de salud incrementaban los riesgos a desarrollar UPP .

En el estudio de Mancini y Onyaguren se determinó que se considera como factores de riesgo para el desarrollo de UPP, la edad y una calificación de riesgo según la escala de Norton de Alta y muy alta (5).

Con respecto a la evaluación de riesgo en la dimensión estado físico, se identificó un alto porcentaje de pacientes $(68.89 \%)$, fueron calificados en un estado físico regular, siendo mayormente mayores de edad en un (85\%) situación lógica pero predisponente la presencia de riesgo.

Cardoso A. concluye a través de un estudio que más del $70 \%$ de las UPP ocurren en mayores de 70 años, y que $60 \%$ de estas se desarrollan en el hospital. Además, mencionó que la incidencia en la población general de úlceras por presión es de $1.7 \%$ entre los 55 y 69 años y de 3.3\% entre los 70 y 75 años de edad. Sin embargo, no existen datos fiables de su incidencia en atención primaria (6). 
Por otro lado en la evaluación del nivel de riesgo en la dimensión estado mental, el $53.64 \%$ de pacientes se encuentran definidos como pacientes en alto riesgo y con estado mental apático, siendo más frecuente en mujeres. Considerando que las características del estado apático es la pérdida de motivación, voluntad, interés hacia las cosas y falta de respuesta afectiva, es sustentable esta situación, basada en los efectos de la crisis de la hospitalización donde la separación del paciente del núcleo familiar va a generar en él un sentimiento de pérdida que sumado a la duda sobre el proceso de la enfermedad genera sentimientos de este tipo, ocasionando a su vez una depresión en la actividad física lo que condicionaría el incremento de riesgos. Los procesos depresivos son muy frecuentes, sobre todo en el paciente de sexo femenino dada su naturaleza, la cual se incrementa en el enfrentamiento con la enfermedad.

En la evaluación de riesgo dimensión actividad física nuevamente se encuentra que el nivel de riesgo alto está determinada por un $67.55 \%$ de la población total, de los cuales se encontró que en su mayoría $(65.56 \%)$ la población estaba en condición de actividad muy limitada. Siendo la actividad física una de las determinantes de mayor relevancia en el desarrollo de úlceras por presión, hecho relacionado con la edad y proceso de enfermedad. La limitación de movimiento debe ser sustituido por el cuidado que permite la sustitución del mismo, siendo probablemente la dimensión donde la responsabilidad profesional adquiere un valor inmensurable.

El Estado Físico General, se determina mediante la evaluación de la nutrición, ingesta de líquidos, temperatura, hidratación, estado de la piel y mucosas.

En el estudio de Jiménez I. "Beneficio de la aplicación de la escala de NORTON en pacientes graves en la Unidad de Cuidados Intensivos. Hospital Militar Docente Dr. Mario Muñoz Monroy. Matanzas. Febrero-diciembre. 2009", donde el uso de la escala de NORTON permitió identificar el nivel de riesgo a presentar UPP de los enfermos ingresados en cuidados intensivos y se concluyó que el $85 \%$ de los pacientes en estudio presentó un nivel de alto riesgo a desarrollar UPP. Por lo tanto, la escala de NORTON ayudó a predecir los pacientes susceptibles a ulcerarse, pues determinó el nivel de riesgo en que se encontraban, precisando el tiempo para una adecuada intervención del personal de enfermería (7).

Se puede explicar estos resultados debido a que la población que entró al estudio tuvo grado de dependencia III en la valoración de enfermería, que implica que el paciente no participa en su autocuidado, con valoración y monitoreo continuo, con funciones vitales alteradas y medicación parenteral constante.

Con respecto a la evaluación de riesgo en la dimensión movilidad, se encontró que dentro de la población de riesgo alto el $61.59 \%$, permanece en posición sentada la mayor parte del tiempo, generando por ello mayor presión en zona sacre, glútea e interglutea.

Según Espinosa Manriquez, en Las úlceras por presión, son una complicación trágica, pero evitable, de la inmovilidad, (8) existiendo una clara relación entre la gravedad del paciente y el riesgo de presentar úlcera por presión, así como con la mayor incidencia de las mismas

Lobo A, en su estudio "Factores de riesgo en el desarrollo de úlceras por presión y sus implicaciones en la calidad de vida", en el año 2008, tuvo como resultado que la inmovilidad aumenta tres veces más el riesgo de presentar UPP. Finalmente, se concluyó que la calidad de vida en una persona que cursa con UPP es baja, siendo esta entidad más frecuente en el sexo femenino, y estando asociadas a mayor mortalidad, perdida de la calidad de vida y aumento en los costos con los tratamientos (9).

Teniendo que, la actividad física es cualquier movimiento corporal, en el cual hay respuesta muscular y que consume más energía en comparación con el estado de reposo. La movilidad es el moverse o trasladarse de un lugar a otro con movimientos continuos.

En la evaluación de la dimensión incontinencia, la mayor población presento una alto nivel de riesgo donde en su mayoría los pacientes $(52.98 \%)$ presentaban incontinencia permanente, fecal o urinaria.

Estos valores son mayores a los encontrados en un trabajo del Grupo Nacional para el Estudio y Asesoramiento en Úlceras por Presión y Heridas Crónicas, que fue de $40-$ $60 \%$. Esto se debe a que los pacientes de dicho estudio tenían todo tipo de grado de dependencia, mientras que el presente trabajo sólo incluye pacientes con grado de dependencia III, que con mucha frecuencia presentan varias comorbilidades (10).

Se entiende por incontinencia al a incapacidad de contener la evacuación de la orina o las heces. Si el paciente tiene control voluntario de la excreción de orina y heces, solicita el urinario cuando es necesario, 4 puntos; ocasional: perdida involuntaria de orina heces, dificultad para solicitar el urinario, requiere de apoyo con sonda vesical, 3 puntos; incontinencia urinaria o fecal: el paciente no controla uno de los dos esfínteres de forma permanente, por su estado clínico tiene una sonda vesical pero está bajo efectos de 
sedación, 2 puntos; doble incontinencia: el paciente no controla ninguno de los esfínteres, tampoco dispone de apoyo de sonda vesical, 1 punto (11).

En la cuantificación de los pacientes que presentaron úlceras por presión durante el seguimiento, se observó que el mayor número de pacientes fue clasificado como Alto riesgo, llegando a desarrollar úlcera 11 de ellos, es decir el $7.28 \%$, seguido del grupo de paciente que tenían un muy alto riesgo (28), desarrollando úlceras por presión 6 pacientes. Teniendo que en los pacientes de muy alto riesgo existía colateralidad mórbida, sustentado en procesos de enfermedad discapacitante producto de alteraciones metabólicas, neurológicas, traumatología, la probabilidad al desarrollo de UPP era un hecho esperado.

Según lo encontrado por Valero, Los pacientes que tenían riesgo alto según Braden fueron quienes más desarrollaron las UPP, así como quienes tenían mayor presencia de enfermedades crónicas y edad de 64 o más años (12).

Carrasco M. (2009), por otro lado afirma en su investigación "Ulceras por presión" que la frecuencia de las UPP aumenta mientras más edad tenga el paciente. Asimismo, concluye que la aparición de las UPP también depende del tipo de institución en la cual se encuentre el paciente, produciéndose cerca de dos tercios de ellas en los hospitales y del resto una parte en las residencias y una menor proporción en sus propios hogares. De todas ellas, más o menos un $2 \%$ son muy severas y pueden comprometer la vida del enfermo (13).

Peralta en el estudio "Frecuencia de casos de UPP en los adultos mayores hospitalizados en el servicio de medicina de un hospital general", realizado en el año 2009, se concluyó que la frecuencia de casos fue de 11,4\%., deduciendo una mejor situación para los pacientes en riesgo probablemente en respuesta a las estrategias aplicadas en los pacientes en riesgo. (14)

\section{REFERENCIAS BIBLIOGRAFICAS}

1. Zabala J. Prevención de las úlceras por presión como derecho universal. Revista Enfermería 2013; 36 (2): 33.

2. García Fernández F. Escalas de valoración del riesgo de desarrollar úlceras por presión. Pressure ulcer risk assessment scales. 2008; 19 (3). Disponible en: http:// dx.doi.org/10.4321/S1134-928X2008000300005

3. Grupo Nacional para el Asesoramiento en Ulceras por Presión y Heridas Crónicas. Escalas e instrumentos de valoración de desarrollar úlceras por presión. Documento Técnico GNEAUPP N XI. 2009.
4. Izquierdo F. Relación entre riesgo e incidencia de úlceras por presión en el adulto mayor hospitalizado en el servicio de medicina del Hospital Nacional Cayetano Heredia 2005. [citado 15 Junio 2011]. Disponible en: http://sisbib.unmsm.edu.pe/bibvirtualdata/monografias/salud/izquierdo_mf/cap1.pdf

5. Mancini P, Oyanguren R. Factores de riesgo para el desarrollo de úlceras por presión en adultos mayores hospitalizados. Hospital Nacional Almanzor Aguinaga Asenjo (HNAAA, 2012, publicado en el 2014 [citado enero 2015]. Disponible en : http://tesis.usat.edu.pe/ jspui/handle/123456789/190

6. Cardoso A. Ulceras por presión. [citado 3 Junio 2011]. Disponible en: http://web.usal.es/ acardoso/temas/ ulceras.html

7. Jiménez I, Gálvez D, De la Rosa H. Beneficio de la aplicación de la escala de Norton en pacientes graves. Unidad de Cuidados Intensivos. Hospital Militar Docente Dr. Mario Muñoz Monroy. Matanzas. Febrerodiciembre. 2009. Cuba. [citado 15 Junio 2011]. Disponible en:

http://www.revmatanzas.sld.cu/revista $\% 20$ medica/ ano $\% 202010 /$ vol5\%202010/tema02.htm

8. Espinoza Manrique E. Ulceras por presión. Disponible en:

www.facmed.unam.mx/deptos/salud/censenanza/spivsa/.../ulceras.pdf

9. Lobo A. Factores de riesgo en el desarrollo de úlceras de presión y sus implicaciones en la calidad de vida. Rev. Bras. Geriatr. Gerontol; 11(3):405-418, [citado en 6 Mayo 2011]. Disponible en: http://bases.bireme. br/cgibin/wxislind.exe/iah/online/?IsisScript=iah/iah. xis $\&$ src $=$ google \&base $=$ LILACS\&lang $=$ p\&nextActio $\mathrm{n}=\mathrm{lnk} \&$ exprSearch $=518205 \&$ indexSearch=ID\#

10. Grupo Nacional para el Asesoramiento en Ulceras por Presión y Heridas Crónicas. Escalas e instrumentos de valoración de desarrollar úlceras por presión. Documento Técnico GNEAUPP N XI. 2009.

11. Ortega M, Suarez M. Manual de evaluación de la calidad del servicio en enfermería. 2da Edición. México. [citado en 09 Abril 2013]. Disponible en: http://books.google. com.pe/books?id=Xgh0A9bF6YMC\&pg=PA84\&dq= Aplicacion + de + la + escala + de + norton $\& \mathrm{hl}=\mathrm{es} \& \mathrm{sa}=\mathrm{X} \&$ ei=ZqRcUdbAG-GligKX6YGoBw\&ved=0CCwQ6A EwAA\#v $=$ onepage $\& q=$ Aplicacion $\% 20 \mathrm{de} \% 201 \mathrm{a} \% 20$ escala $\% 20 \mathrm{de} \% 20$ norton $\& \mathrm{f}=$ false.

12. Valero H, Parra D, Gómez R, Camargo-Figuera F. 
Prevalencia de úlcera por presión en una institución de salud Rev. Univ. Ind. Santander. Salud. 2011; 43 (3). Disponible en: www.scielo.org.co/scielo. php?pid=S0121-08072011000300005

13.Carrasco M, Ramallo W, Hernández G. Ulceras por presión. Argentina: Editorial el Cid, 2009.

14.Peralta C, Varela L, Gálvez M. Frecuencia de casos de úlceras de presión y factores asociados a su desarrollo en adultos mayores hospitalizados en los servicios de Medicina de un hospital general. Rev Med Hered. 2009; 20 (1): 16-1. [citado 5 Mayo 2011]. Disponible en:http://www.scielo.org.pe/scielo.php?script=sci_artt ext\&pid=S1018130X2009000100005\&lng=es\&nrm= iso $>$. ISSN 1018-130X.

Fecha de recibido: 12 de Octubre de2014

Fecha de aceptación: 26 de Noviembre de 2014. 